\title{
Xylella fastidiosa Plasmid-Encoded PemK Toxin Is an Endoribonuclease
}

\author{
Min Woo Lee, Elizabeth E. Rogers, and Drake C. Stenger
}

San Joaquin Valley Agricultural Sciences Center, United States Department of Agriculture-Agricultural Research Service, Parlier, CA 93648.

Accepted for publication 16 July 2011

\section{ABSTRACT}

Lee, M. W., Rogers, E. E., and Stenger, D. C. 2012. Xylella fastidiosa plasmid-encoded PemK toxin is an endoribonuclease. Phytopathology $102: 32-40$

Stable inheritance of pXF-RIV11 in Xylella fastidiosa is conferred by the pemI/pemK toxin-antitoxin (TA) system. PemK toxin inhibits bacterial growth; PemI is the corresponding antitoxin that blocks activity of PemK by direct binding. PemK and PemI were overexpressed in Escherichia coli and activities of each were assessed. Purified PemK toxin specifically degraded single-stranded RNA but not double-stranded RNA, double-stranded DNA, or single-stranded DNA. Addition of PemI antitoxin inhibited nuclease activity of PemK toxin. Purified complexes of PemI bound to PemK exhibited minimal nuclease activity; removal of
PemI antitoxin from the complex restored nuclease activity of PemK toxin. Sequencing of $5^{\prime}$ rapid amplification of cDNA ends products of RNA targets digested with PemK revealed a preference for cleavage between $U$ and A residues of the sequence UACU and UACG. Nine single amino-acid substitution mutants of PemK toxin were constructed and evaluated for growth inhibition, ribonuclease activity, and PemI binding. Three PemK point-substitution mutants (R3A, G16E, and D79V) that lacked nuclease activity did not inhibit growth. All nine PemK mutants retained the ability to bind PemI. Collectively, the results indicate that the mechanism of stable inheritance conferred by pXF-RIV11 pemI/pemK is similar to that of the R100 pemI/pemK TA system of E. coli.

Additional keywords: Pierce's disease, plasmid shuttle vector.
Bacteria encode a variety of toxin-antitoxin (TA) systems that serve as growth regulators consisting of a stable toxin and a cognate labile antitoxin. TA systems are grouped into at least eight families based on sequence and functional similarity $(21,29)$. Toxin mode of action varies among families: growth inhibition or cell death may result from kinase activity (9), inhibition of DNA replication $(4,14,26)$. or, most commonly, degradation of cellular RNA $(2,5,7,22,23,39,40)$. Antitoxins may be proteins that bind toxins (18), antisense RNA repressing toxin expression (11), or RNA that directly interacts with toxin protein (10). TA systems resident on bacterial chromosomes induce programmed cell death in response to specific conditions (15), including biofilm formation (16) and various environmental stresses $(6,7,12,21,28)$. When encoded by plasmids, TA systems serve as plasmid addiction systems in which residual stable toxin in plasmid-free daughter cells results in postsegregational killing $(1,13,20,25$, $26,36,37)$. In some cases, TA systems result in exclusion of competing plasmids (8).

Xylella fastidiosa is the causal agent responsible for Pierce's disease of grape and other vascular diseases of horticultural and landscape plants (38). In nature, X. fastidiosa is limited to the xylem of infected plants or the foregut of xylem-feeding insects that transmit the bacterium (3). Confirmation of $X$. fastidiosa pathogenicity or virulence determinants deduced from mutant phenotypes may be accomplished by complementation assays. Matsumoto et al. (19) developed a complementation system in which suicide plasmids deliver wild-type versions of mutated genes to a neutral site in the $X$. fastidiosa chromosome. Recently,

Corresponding author: D. C. Stenger; E-mail address: drake.stenger@ars.usda.gov

http://dx.doi.org/10.1094/PHYTO-05-11-0150

This article is in the public domain and not copyrightable. It may be freely reprinted with customary crediting of the source. The American Phytopathological Society, 2012. a plasmid shuttle vector has been developed for $X$. fastidiosa based on replication and stability modules derived from pXFRIV11 (17), an $\approx 25$-kbp plasmid recovered from mulberryinfecting strains of $X$. fastidiosa (32). Phylogenetic analysis of the replication initiator protein TrfA indicated that pXF-RIV11 belongs to a newly recognized and highly divergent clade of incompatibility group P-1 plasmids (31). For use in complementation assays conducted in planta, shuttle vectors for $X$. fastidiosa need to be stable in the absence of antibiotic selection. Although plasmids bearing the replication module (trfA and the origin of replication) of pXF-RIV11 could propagate in $X$. fastidiosa, culturing in the absence of antibiotic selection resulted in loss of the plasmid after three serial passages conducted at weekly intervals (17). Adding the pemI/pemK TA system homologue from pXF-RIV11 conferred stable inheritance of the shuttle vector in the absence of antibiotic selection. PemK was validated as a toxin inhibiting bacterial growth; PemI was shown to be the cognate antitoxin that prevented growth inhibition by direct binding to PemK toxin (17). However, the mechanism by which pXF-RIV11 PemK toxin inhibits bacterial cell growth has not been determined. In this report, we characterize ribonuclease activity of pXF-RIV11 PemK and show that PemK mutants lacking nuclease activity do not inhibit cell growth, thereby demonstrating that the mechanism of pXF-RIV11 PemK toxicity is similar to that of PemK homologues encoded by plasmid R100 of Escherichia coli (40) and the chromosome of Bacillus anthracis (2).

\section{MATERIALS AND METHODS}

Protein expression, purification, and Western blot analysis. E. coli strain BL21(DE3)pLysS (Stratagene, La Jolla, CA) was used for all protein expression experiments. Plasmid constructs for individual expression of pXF-RIV11 PemK or PemI and dual expression of both proteins has been described (17) using the 
vectors pET28a (His-tagged PemK only), pCDF1b (His-tagged PemI only), and pETDuet-1 (both His-tagged PemK and S-tagged PemI). Additional plasmids for expression of PemK mutants were constructed as described below using the expression vectors pET28a (His-tagged PemK only) or pETDuet-1 (both His-tagged PemK and S-tagged PemI). For simplicity, tags present on expressed proteins are referred to below only when directly relevant to interpretation of experimental results.

For extraction of recombinant proteins, expression was induced at an optical density at $600 \mathrm{~nm}\left(\mathrm{OD}_{600}\right)$ of 0.6 by adding $1 \mathrm{mM}$ isopropyl- $\beta$-D-thiogalactopyranoside (IPTG); cultures were harvested $4 \mathrm{~h}$ after induction. His-tagged proteins were purified from soluble cell extracts using Ni-nitrilotriacetic acid (NTA) affinity chromatography as described by the manufacturer (Qiagen, Valencia, CA). Purified proteins recovered from Ni-NTA columns were dialyzed in $50 \mathrm{mM}$ glycine and $5 \mathrm{mM}$ EDTA, pH 9.0, and stored frozen at $-20^{\circ} \mathrm{C}$. Purified protein concentration was estimated by measuring absorbance at $280 \mathrm{~nm}$ using an ND-1000 spectrophotometer (NanoDrop, Wilmington, DE) and the default extinction coefficient of $1.0 \mathrm{mg} / \mathrm{ml}$. Protein electrophoresis (sodium dodecyl sulfate polyacrylamide gel electrophoresis [SDS-PAGE]) and Western blot assays were performed using standard methods (27); samples were denatured by boiling in the presence of SDS and $\beta$-mercaptoethanol. Primary antibodies, anti-His (Calbiochem, San Diego, CA) and anti-S (Novagen, La Jolla, CA), were used at a 1:2,000 dilution; horseradish peroxidase-conjugated goat anti-mouse secondary antibody (Santa Cruz Biotechnology, Santa Cruz, CA) was used at a 1:15,000 dilution and detected using a Fast ECL kit (Thermo Scientific Pierce, Waltham, MA).

Complexes of S-tagged PemI bound to His-tagged PemK were recovered by Ni-NTA chromatography. To remove S-tagged PemI from complexes containing His-tagged PemK, proteins were dissociated in the presence of $5 \mathrm{M}$ guanidine-HCl. Denatured Histagged PemK was selectively captured by Ni-NTA chromatography, eluted from the column, allowed to refold during dialysis in $50 \mathrm{mM}$ glycine and $5 \mathrm{mM}$ EDTA, $\mathrm{pH} 9.0$, and stored at $-20^{\circ} \mathrm{C}$.

Nuclease assays. Single-stranded RNA (ssRNA) substrate used in nuclease assays was total RNA extracted from young leaves of Nicotiana tabacum using TRIzol reagent (Life Technologies, Foster City, CA) following the manufacturer's protocol. Alternative substrates consisted of double-stranded RNA (dsRNA), double-stranded DNA (dsDNA), and single-stranded DNA (ssDNA). Homalodisca vitripennis reovirus dsRNA was purified from infected glassy-winged sharpshooters by CF11 chromatography as described (33). DNA substrates were from commercially available sources: the Invitrogen 1-kb Plus DNA ladder (dsDNA) or m13MP18 virion DNA (ssDNA) from New England Biolabs. For some assays, RNaseA (Invitrogen, Carlsbad, CA) or RQ1 DNase (Promega Corp., Madison, WI) were included as controls. Nuclease assays were conducted by incubation (10 min at room temperature) of nucleic acid substrate ( $5 \mu \mathrm{g}$ of ssRNA or $2 \mu \mathrm{g}$ of other substrates) with purified PemK $(0.25 \mu \mathrm{g})$ in a $20-\mu \mathrm{l}$ volume. Inhibition of PemK nuclease activity by PemI was assessed by mixing purified PemI $(0.4,1.0$, or $2.0 \mu \mathrm{g})$ with purified PemK $(0.25 \mu \mathrm{g})$ for $10 \mathrm{~min}$ prior to adding the mixture to ssRNA substrate. Products were evaluated by electrophoresis in $1.4 \%$ agarose-formaldehyde (ssRNA), $1.5 \%$ agarose (dsRNA), or $1 \%$ agarose (dsDNA and ssDNA) gels and visualized by staining with ethidium bromide. The 1-kb RNA ladder (Invitrogen) was used as a size standard for ssRNA samples; other substrates were of known size, such that separate size standards were not needed.

Determination of PemK cleavage site preference. Three ssRNA targets were digested with PemK; products of each were used as templates in $5^{\prime}$ rapid amplification of cDNA ends (RACE) reactions. Reaction conditions employed for 5' RACE were as described (34). Target RNAs included N. tabacum chloroplast 23S rRNA (GenBank accession number AY123764) present in total RNA samples and two synthetic RNAs generated by in vitro transcription using the T7 MEGAscript kit (Ambion, Austin, TX), as described (30). The synthetic RNAs corresponded to 1,251 nucleotides of the $H$. coagulata virus 1 (HoCV-1) RNA 5' terminus (GenBank accession number DQ288865) or nucleotides 64 to 1,316 of the $X$. fastidiosa cys $G$ coding region (GenBank accession number CP000941). Both in vitro transcripts also included 5'- or 3'-proximal sequences derived from pGEMT-Easy (cysG) or
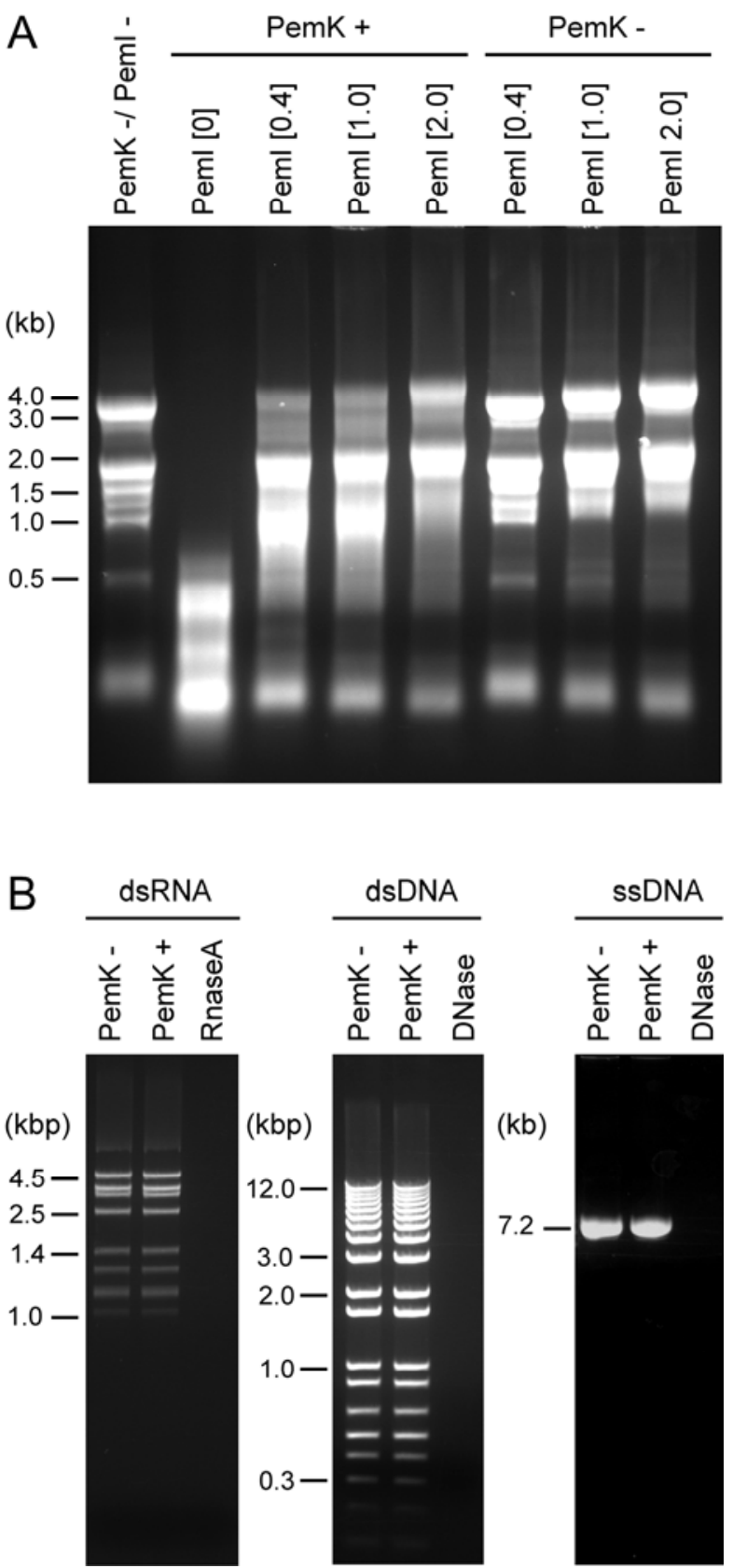

Fig. 1. PemK toxin is a single-stranded ribonuclease inhibited by PemI antitoxin. A, Electrophoresis of Nicotiana tabacum RNA samples following incubation with $0.25 \mu \mathrm{g}$ of PemK $(\mathrm{PemK}+)$ or no PemK $(\mathrm{PemK}-)$ toxin and various amounts $(0,0.4,1.0$, or $2.0 \mu \mathrm{g})$ of PemI antitoxin. B, Electrophoresis of Homalodisca vitripennis reovirus double-stranded RNA (dsRNA), Invitrogen 1-kb Plus ladder double-stranded DNA (dsDNA), or M13mp18 single-stranded DNA (ssDNA) samples following incubation with $0.25 \mu \mathrm{g}$ of PemK $(\mathrm{PemK}+)$ or no $(\mathrm{PemK}-)$ toxin. As controls, samples were digested with RNaseA (dsRNA) or DNase (dsDNA and ssDNA). Sizes are indicated at left in kilobase pairs (kbp) for double-stranded molecules or in kilobases $(\mathrm{kb})$ for single-stranded molecules. 
pUC19 (HoCV-1). For all three target sequences, nested genespecific primers were selected to anneal $\approx 200$ nucleotides downstream (in plus sense) of the sequence UAC, in what was predicted to be the largest product resulting from PemK digestion of the target RNA if the recognition site specificity of the pXFRIV11 PemK homologue was the same as that determined for PemK encoded by R100 from E. coli (40). Primers used for reverse transcription annealed to $N$. tabacum $23 \mathrm{~S}$ rRNA nucleotides 1,296 to 1,276 , HoCV-1 nucleotides 605 to 584 , or $X$. fastidiosa cysG nucleotides 373 to 354 . The resulting cDNAs were C-tailed using terminal deoxynucleotidyl transferase (TdT); polymerase chain reactin (PCR) was conducted using the AAP polyG/I primer (Invitrogen) and gene-specific primers annealing to $N$. tabacum $23 \mathrm{~S}$ rRNA nucleotides 1,282 to 1,260 , HoCV-1 nucleotides 587 to 566 , or $X$. fastidiosa cys $G$ nucleotides 352 to 334. PCR products were ligated into pGEMT-easy and transformed into E. coli JM109 competent cells (Promega Corp.). Sequences were determined for inserts of $5^{\prime}$ RACE clones and aligned with the respective target sequence. The $5^{\prime}$-proximal base adjacent to the homopolymeric $G$ track (corresponding to the

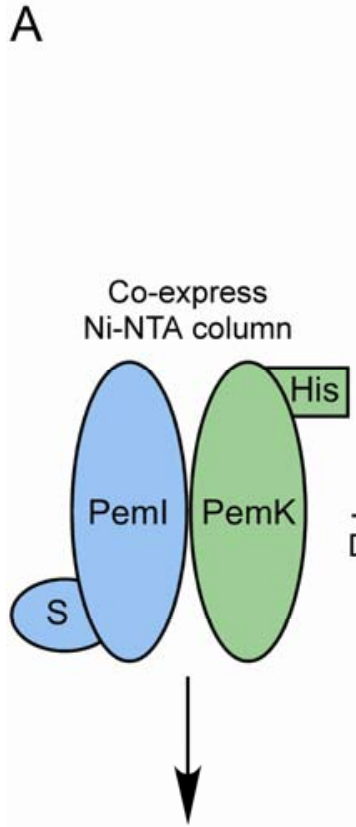

Lane 2
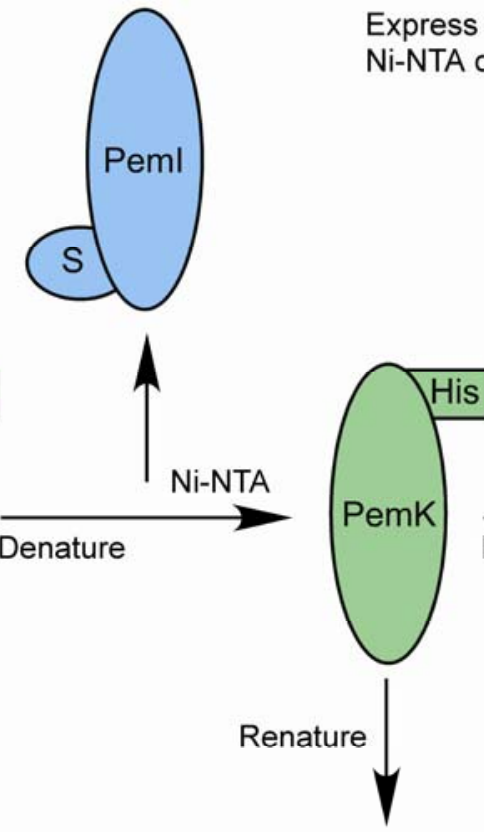

Lane 3
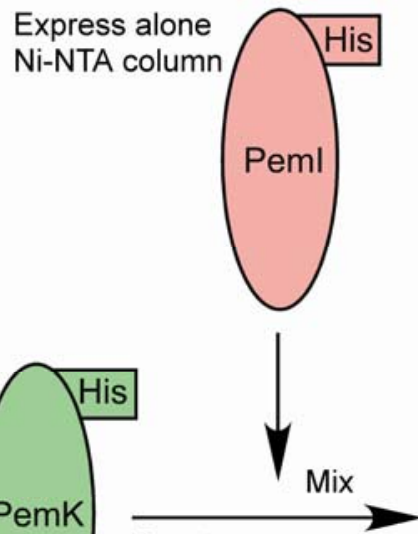

Renature

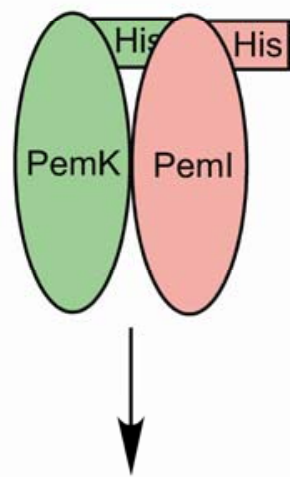

Lane 4
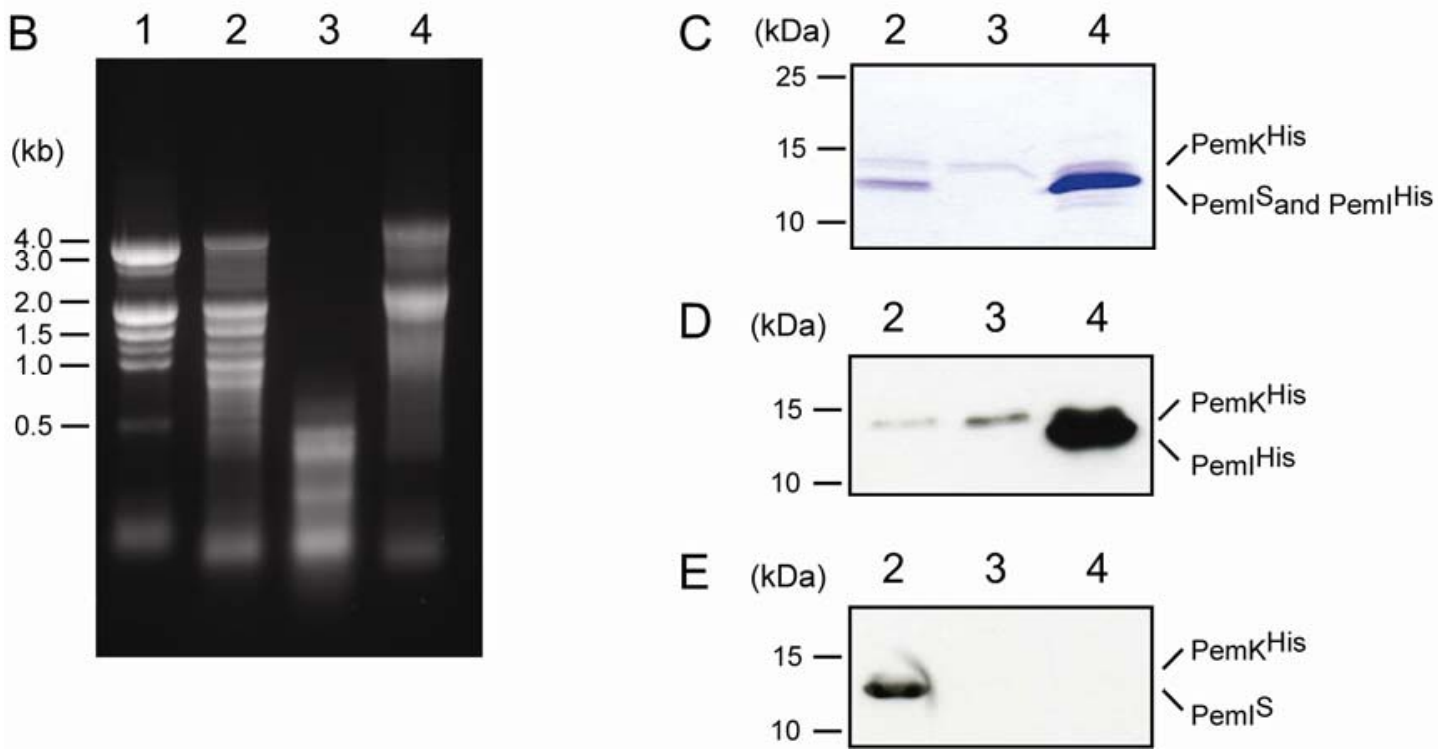

Fig. 2. Purification and activity of PemK toxin complexed with PemI antitoxin. A, Coexpression of His-tagged PemK (PemK $\left.{ }^{H i s}\right)$ and S-tagged PemI (PemI ${ }^{S}$ ) results in recovery of $\mathrm{PemI}^{\mathrm{S}} / \mathrm{PemK}^{\mathrm{His}}$ complex by Ni-nitrilotriacetic acid (NTA) chromatography (lane 2) due to specific binding of PemI to PemK. PemI ${ }^{\mathrm{S}}$ was removed from the complex by denaturation; PemK ${ }^{\text {His }}$ was purified by a second cycle of Ni-NTA chromatography and renatured (lane 3 ). PemI ${ }^{\text {His }}$ was expressed alone, purified by Ni-NTA chromatography, and added back to renatured PemK ${ }^{\text {His }}$ (lane 4). B, Ribonuclease assays of Nicotiana tabacum RNA incubated with no

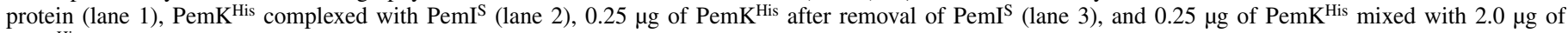
$\mathrm{PemI}^{\text {His }}$ (lane 4). Mobility of RNA size standards indicated at left in kilobases (kb). C, Sodium dodecyl sulfate polyacrylamide gel electrophoresis (Coomassie stained) of proteins recovered at various stages of purification. D, Western blot of purified proteins probed with His antibodies. E, Western blot of purified proteins probed with $\mathrm{S}$ antibodies. Lane assignments in $\mathrm{C}$ to $\mathrm{E}$ are as designated in A; sizes of protein standards indicated at left in kilodaltons (kDa); identity of proteins designated at right. 
region tailed by TdT) represents the terminal residue of each RNA template from which individual clones were reverse transcribed. Sites preferentially cleaved by PemK were identified based on the occurrence of identical $5^{\prime}$ terminal residues in multiple clones sequenced for each 5' RACE reaction.

Because initial 5' RACE reactions with the three RNA targets described above indicated PemK cleaved within UACU but not UACA, two additional 5' RACE reactions (RACE 2 and RACE 3) were conducted with PemK digested in vitro transcripts of $X$. fastidiosa cysG, with primers positioned downstream of UACA (nucleotides 779 to 782) or UACC (nucleotides 966 to 969), respectively. Primers used for reverse-transcription annealed to $X$. fastidiosa cys $G$ nucleotides 902 to 882 (cysG RACE 2) or 1,109 to 1,087 (cys $G$ RACE 3). Gene-specific primers used for PCR annealed to $X$. fastidiosa cys $G$ nucleotides 886 to 867 (cys $G$ RACE 2) or 1,091 to 1,073 (cysG RACE 3).

Generation of PemK mutants. Single substitution mutations in the 5'-proximal coding region of PemK were generated by PCR using various forward primers altered at one codon position and a wild-type reverse primer. Mutant primers were designed to generate the mutations R3A, G4A, D5A, D5E, P13A, H17A, and H22A. Two additional single-substitution mutants (G16E and D79V) were generated by random PCR mutagenesis (35). PCR products were cloned into pGEMT-easy, each mutation was verified by sequencing, and mutated pem $K$ sequences used to replace the wild-type pem $K$ sequence in the expression vectors described above.

Growth inhibition assays. Cultures (three replicates per treatment) were grown overnight at $37^{\circ} \mathrm{C}$ with shaking in LuriaBertani (LB) media containing chloramphenicol at $50 \mu \mathrm{g} / \mathrm{ml}$ and kanamycin at $50 \mu \mathrm{g} / \mathrm{ml}$. The cultures were then diluted to $\mathrm{OD}_{600}=$ 0.1 in fresh LB medium with antibiotics and grown at $37^{\circ} \mathrm{C}$ with shaking to $\mathrm{OD}_{600}=0.3$, and protein expression was induced by addition of IPTG to $1 \mathrm{mM}$. Cultures expressing wild-type PemK, mutant PemK, or no protein (empty vector) were monitored for growth by measuring $\mathrm{OD}_{600}$ hourly for $5 \mathrm{~h}$. Statistical significance of differences in growth were determined for the 5-h time point by analysis of variance $(P<0.0001)$ and Tukey-Kramer highly significant difference multiple comparison $(P=0.01)$ tests.

\section{RESULTS}

PemK is an endonuclease specific for ssRNA and is inhibited by PemI. Nuclease assays were conducted using purified PemK toxin with or without PemI antitoxin (Fig. 1). In the absence of PemI, PemK degraded N. tabacum total RNA to products $<600$ bases in $10 \mathrm{~min}$ at room temperature (Fig. 1A). Increasing incubation time did not alter electrophoretic mobility of digestion products (data not shown). Addition of increasing amounts of PemI to assays containing PemK resulted in correspondingly less

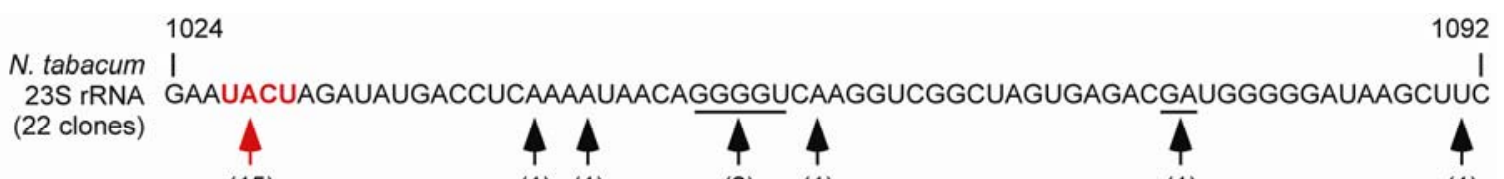

(15)

(1) (1)

(2) (1)

(1)

(1)

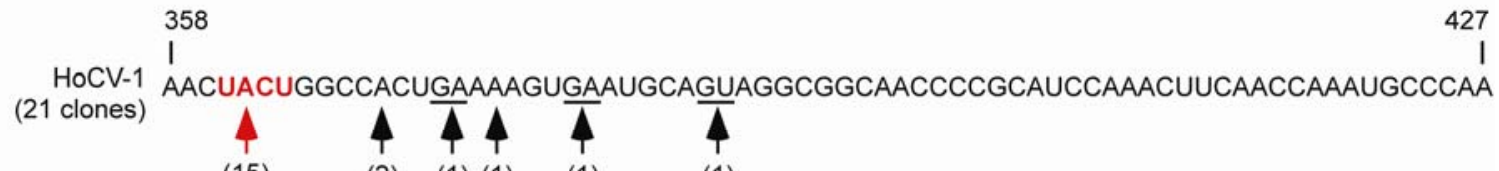

(15)

(2) (1) (1)

(1)

(1)

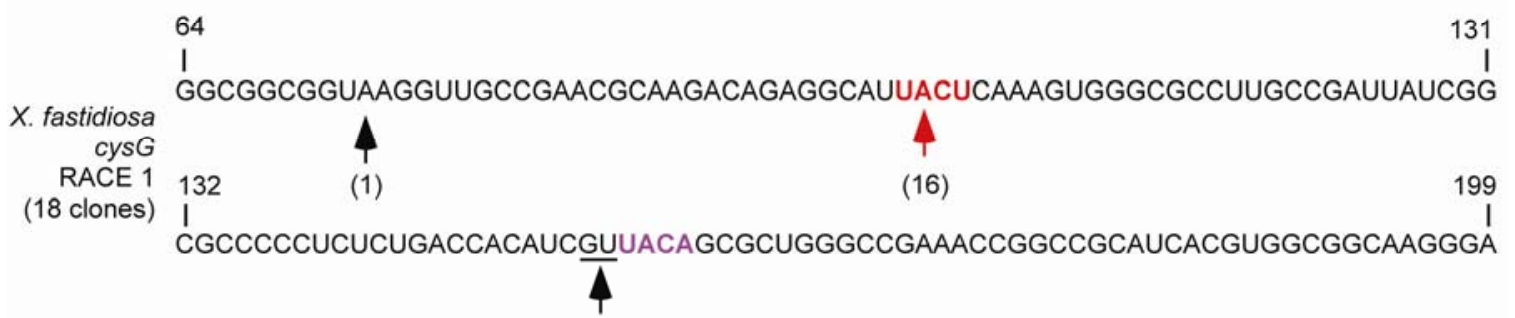

(1)
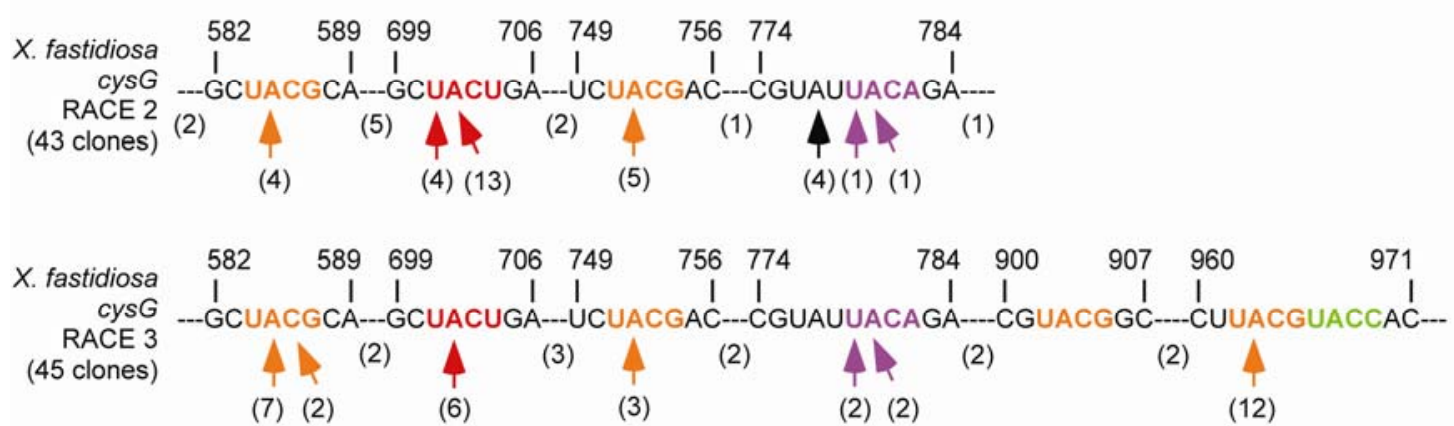

Fig. 3. Cleavage site preference of PemK ribonuclease. Presented are results of 5' rapid amplification of cDNA ends (RACE) reactions conducted using RNA templates previously digested with pXF-RIV11 PemK. RNA substrates used were Nicotiana tabacum 23S rRNA or synthetic T7 transcripts of Homalodisca coagulata Virus 1 (HoCV-1) RNA and the cysG coding region of Xylella fastidiosa. Three separate 5' RACE reactions (RACE 1, RACE 2, and RACE 3) were conducted with PemK-digested $X$. fastidiosa cys $G$ RNA template using different gene-specific primers. Terminal $5^{\prime}$ residues (arrows) were determined by sequencing clones of 5' RACE products; parentheses indicate number of clones with identical termini. Colors indicate location of UACN sites. Note that multiple clones had 5' termini mapping to the A residue of UAC followed by $\mathrm{U}$ or $\mathrm{G}$ but not $\mathrm{C}$ and rarely A. Underline denotes termini imprecisely mapped due to addition of homopolymeric tail added to $5^{\prime}$ end of cDNA. 
degradation of $N$. tabacum total RNA. Minimal degradation of $N$. tabacum total RNA was noted in assays containing increasing amounts of PemI but lacking PemK. Due to addition of various amounts of purified proteins stored in $50 \mathrm{mM}$ glycine and $5 \mathrm{mM}$ EDTA, pH 9.0, reaction conditions varied in concentration of glycine $(12.5$ to $37.5 \mathrm{mM})$ and EDTA (1.75 to $3.75 \mathrm{mM})$. However, test reactions indicated that variation in concentrations of glycine and EDTA (over the ranges stated above) did not affect ribonuclease activity of PemK (data not shown) but did result in minor changes in electrophoretic mobility of RNA (Fig. 1A). To assess specificity of PemK nuclease activity, alternative nucleic acid targets were incubated with PemK. Electrophoretic mobility of dsRNA, dsDNA, and ssDNA was unaffected by incubation with PemK (Fig. 1B). These results indicate that nuclease activity of PemK is specific for ssRNA, PemI inhibits nuclease activity of PemK, and PemK is an endoribonuclease that likely recognizes specific sequences for cleavage.

Expression of PemK in E. coli is problematic (e.g., low yields) due to inhibition of bacterial growth by PemK (17). Therefore, PemK was coexpressed with PemI. Although PemK was Histagged and PemI was S-tagged, both proteins are recovered in the eluate of Ni-NTA columns due to specific binding of PemI to PemK (17). Nuclease assays (Fig. 2) using the purified PemI/ PemK complex resulted in only limited degradation of $N$. tabacum total RNA. S-tagged PemI was removed from the complex by denaturation and a second cycle of Ni-NTA chromatography (Fig. 2A). Upon renaturation, His-tagged PemK digested substrate RNA to products of $<600$ bases, indicating full restoration of endoribonuclease activity (Fig. 2B). Subsequent addition of PemI (expressed separately as a His-tagged protein) reduced nuclease activity of PemK but RNA substrate was slightly more degraded compared with substrate incubated with co-purified PemI/PemK complex. This difference may be due to lack of complete binding of PemI added back to PemK. Presence or absence of PemI and PemK at various stages in the experiment described above was verified by SDS-PAGE and Western blots using antibodies specific to the His- and S-tags (Fig. 2C to E). These results indicate that inhibition of PemK nuclease activity by PemI requires binding and is reversible.

PemK cleaves a specific RNA sequence. RNaseA preferentially cleaves phosphodiester bonds on the $5^{\prime}$ side of pyrimidine residues (24), such that complete digestion of most natural RNA

A
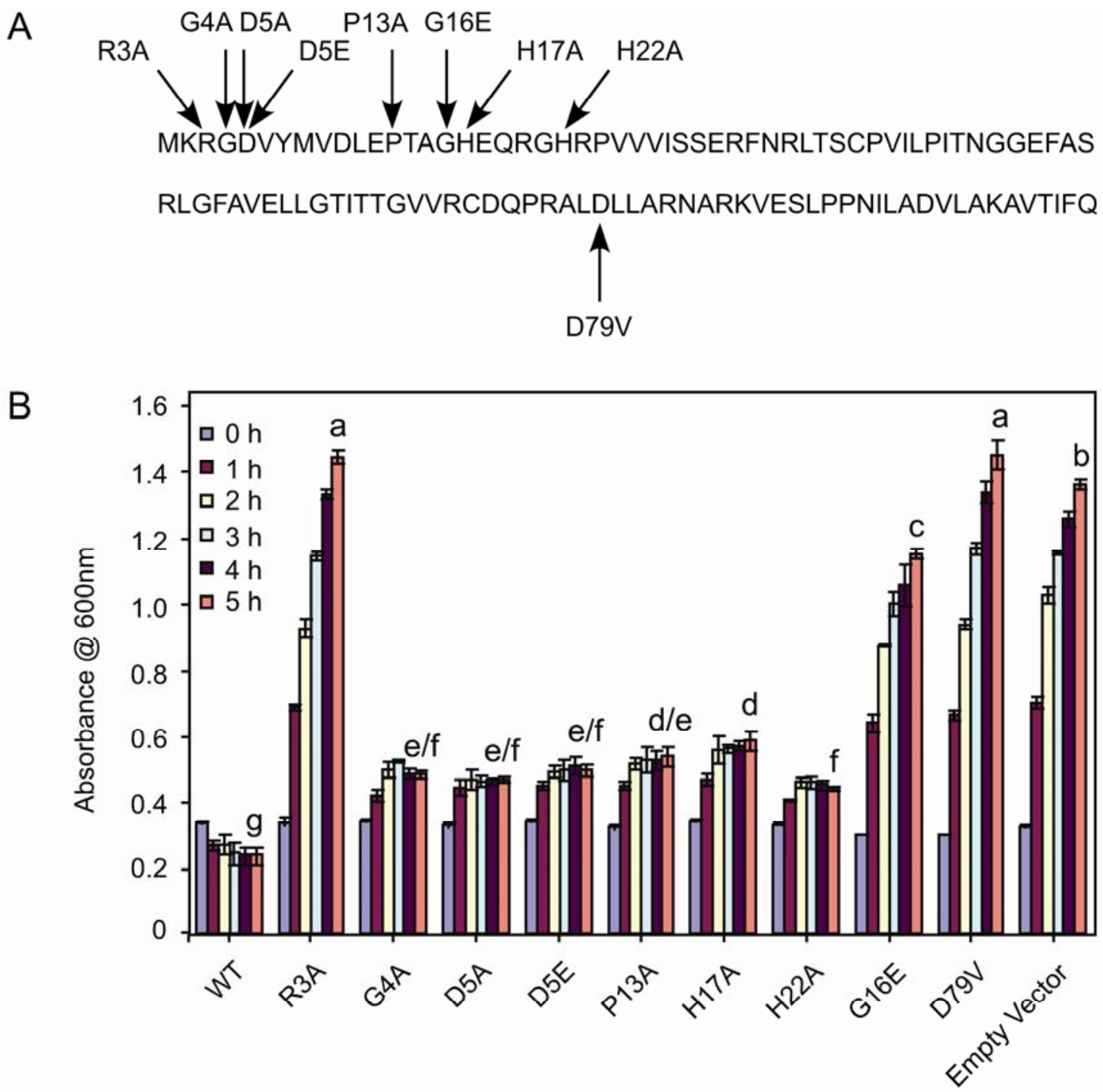

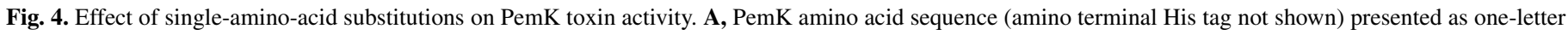

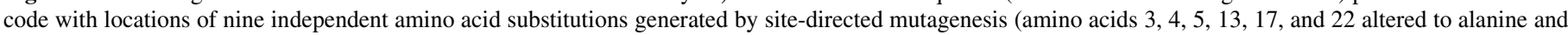

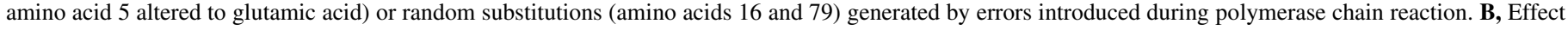

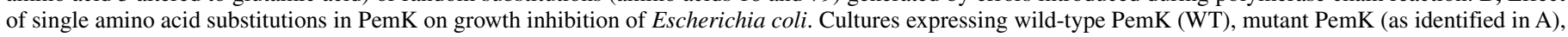

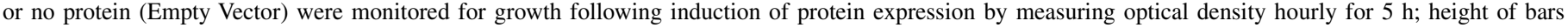

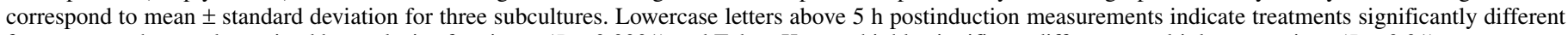
from one another, as determined by analysis of variance $(P<0.0001)$ and Tukey-Kramer highly significant difference multiple comparison $(P=0.01)$ tests. 
substrates results in short products. In contrast, PemK digestion of $N$. tabacum total RNA yielded products up to $\approx 600$ bases in length (Figs. 1 and 2), suggesting that PemK has sequence specificity requirements more constrained relative to RNaseA. To determine sites preferentially recognized and cleaved by PemK, 5' RACE was conducted on three target RNAs following digestion with PemK: native $N$. tabacum 23S rRNA (present in total RNA samples) and synthetic T7 transcripts of HoCV-1 or X. fastidiosa cysG RACE 1 (Fig. 3). Following PCR, 5' RACE products were composed of a single band of $\approx 200$ to $250 \mathrm{bp}$ for each target RNA. Sequences of cloned 5' RACE products were determined and aligned with known sequences of the three targets. For all three target RNAs, the majority of cloned 5' RACE products had the A residue of the sequence UACU as the $5^{\prime}$-terminal residue (adjacent to the homopolymeric tail added by TdT), indicating a preference for PemK cleavage between the $U$ and A residues of the recognition sequence UACU. For each RNA target, a minority of 5' RACE clones had 5' termini at various positions, usually downstream of the UACU site. Most alternative $5^{\prime}$ termini occurred only once (or at most twice) in each data set and were assumed to have resulted from premature termination during reverse transcription, although it cannot be excluded that some of these $5^{\prime}$ termini may represent less-preferred PemK cleavage sites. Interestingly, $X$. fastidiosa cys $G$ contained the sequence UACA (nucleotides 155 to 158) located between the primer annealing site and the predominant $5^{\prime}$ terminus obtained in RACE 1. This observation suggests that the pXF-RIV11 PemK recognition site may involve more than UAC. Alternatively, failure to cleave the $X$. fastidiosa cys $G$ sequence UACA could be due to secondary structure, because PemK does not cleave dsRNA (Fig. 1B). However, secondary structure predicted by MFOLD (http:// mfold.rna.albany.edu/) places UACA (nucleotides 155 to 158) completely within a single-stranded bulge (data not shown).
To further assess whether the residue positioned immediately $3^{\prime}$ of UAC affects cleavage of RNA by pXF-RIV11 PemK, two additional 5' RACE reactions (RACE 2 and RACE 3) were conducted using PemK-digested $X$. fastidiosa cys $G$ transcript and alternative gene-specific primer annealing sites (Fig. 3). Gene-specific primers were positioned such that the first upstream UAC was in the context of UACA (nucleotides 779 to 782) for RACE 2 or UACC (nucleotides 966 to 969) for RACE 3. Sequences were determined for 43 clones for RACE 2 and 45 clones for RACE 3. In contrast to 5' RACE reactions for $N$. tabacum 23S rRNA, HoCV-1, and $X$. fastidiosa cysG RACE 1, multiple 5' RACE products were observed upon gel electrophoresis following PCR and no single nucleotide position constituted the majority of $5^{\prime}$ termini for RACE 2 or RACE 3. These results suggested that this region of cysG RNA had not been completely digested with PemK, because complete digestion should have produced a single 5' RACE product dominated by the cleavage site closest to the primer annealing site. Among RACE 2 products, only one clone had a 5'-terminus at A residue 780 within UACA and a second clone terminated at $\mathrm{U}$ residue 779. Among RACE 3 products, no clones terminated within UACC and 5' termini within UACA were limited to two each at $A$ (nucleotide 780) or the 5'-proximal U (nucleotide 779). The most common 5' termini for both RACE 2 and RACE 3 were the A residue of UACU (nucleotides 701 to 704) or UACG (nucleotides 584 to 587, 751 to 754, and [RACE 3 only] 962 to 965). Mfold secondary structure predictions (data not shown) indicated that two sites (UACG at nucleotides 902 to 905 and UACA at nucleotides 779 to 782 ) inefficiently (or not at all) cleaved by PemK were located within stems, whereas two sites (UACG at nucleotides 584 to 587 and UACU at nucleotides 701 to 704) more frequently cleaved by PemK were positioned within loops. However, UACG (nucleotides 751 to 754) was cleaved by PemK, despite being positioned within a stem; and two sequences partially
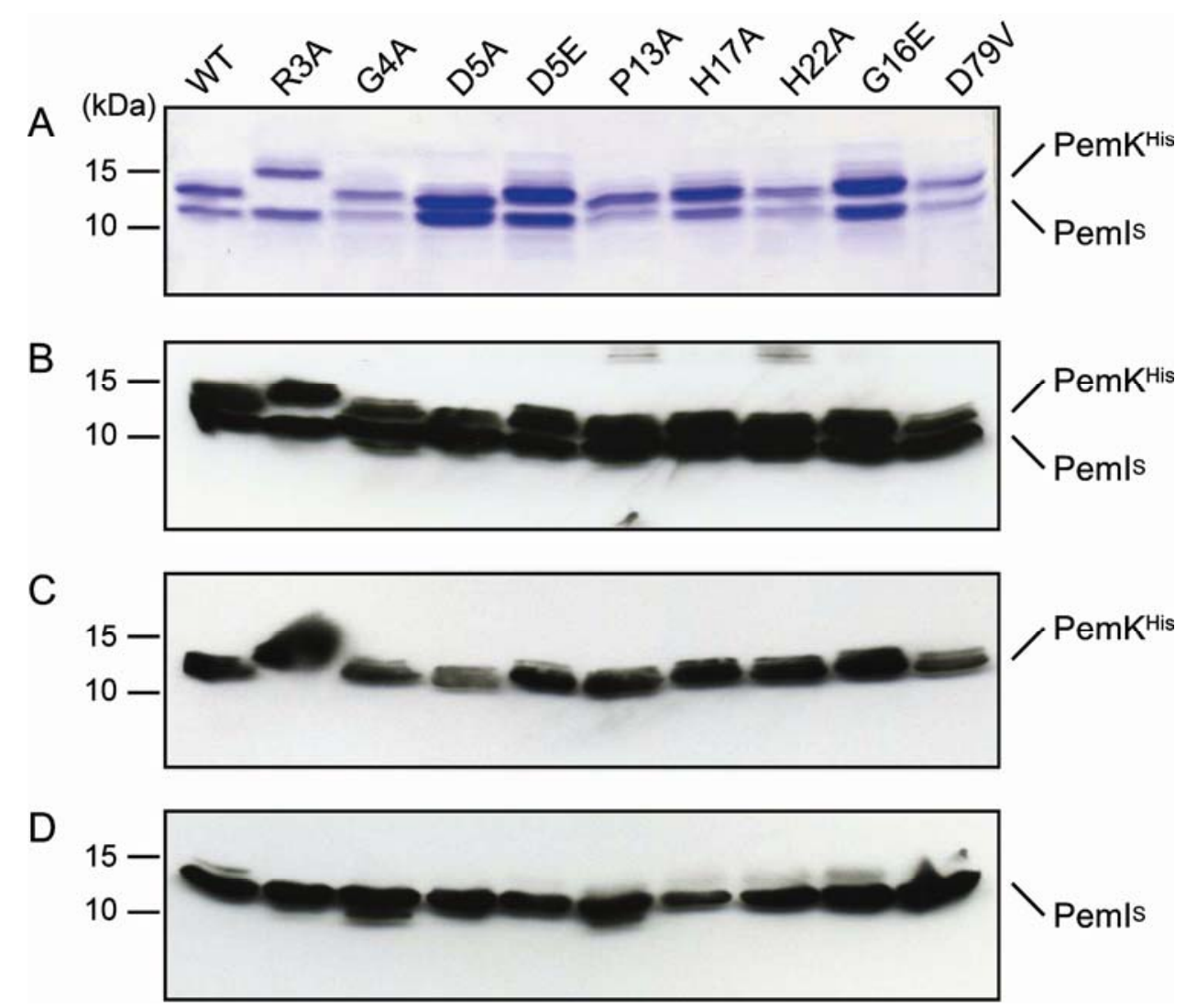

Fig. 5. Binding of PemI to PemK mutants. Wild type (WT) PemK ${ }^{\text {His }}$ and nine single-amino-acid substitution mutants of PemK ${ }^{\text {His }}$ were each coexpressed with $\mathrm{PemI}^{\mathrm{S}}$ and purified by Ni-nitrilotriacetic acid chromatography. Purified proteins were analyzed by A, sodium dodecyl sulfate polyacrylamide gel electrophoresis (Coomassie stained); B, Western blotting using a mixture of His and S antibodies as probe; $\mathbf{C}$, Western blotting using His antibodies as probe; and $\mathbf{D}$, Western blotting using $\mathrm{S}$ antibodies as probe. Note that all PemK mutants retained the ability to bind PemI. 
overlapping stems were either cleaved (UACG at nucleotides 962 to 965 ) or not cleaved (UACC at nucleotides 966 to 969) by PemK. Collectively, the data from all 5' RACE reactions indicate that the sequence UAC is the preferred recognition site for pXF-RIV11 PemK, with efficiency of cleavage influenced by identity of the base (U and G preferred over $\mathrm{C}$ and A) $3^{\prime}$ proximal to UAC. Although secondary structure clearly affects efficiency of cleavage (dsRNA is not a substrate for PemK), in silico predictions of ssRNA folding did not correlate well with efficiency of cleavage by PemK.

PemK nuclease activity is required for growth inhibition. Nine single-amino-acid substitution mutants of PemK were constructed and evaluated for growth inhibition upon expression in E. coli following induction with IPTG (Fig. 4). Expression of wild-type PemK or six substitution mutants (G4A, D5A, D5E, $\mathrm{P} 13 \mathrm{~A}, \mathrm{H} 17 \mathrm{~A}$, and H22A) in the absence of PemI resulted in growth inhibition over $5 \mathrm{~h}$ postinduction compared with the empty vector control. In contrast, growth inhibition was either not observed (R3A and D79V) or minimal (G16E) for three PemK mutants (expressed without PemI) following induction with IPTG. Each PemK mutant (His-tagged) was coexpressed with Stagged PemI and purified by Ni-NTA chromatography; no growth inhibition was noted (data not shown) and all PemK mutants retained the ability to bind PemI, based on co-purification of Stagged PemI with His-tagged PemK by Ni-NTA chromatography (Fig. 5). Loss of growth inhibition phenotype by certain PemK mutants was not due to instability, because PemK protein was detected by SDS-PAGE and western blotting for all nine mutants. One mutant (R3A) exhibited minor variation in electrophoretic mobility compared with that of wild-type PemK.

For use in nuclease assays, PemI was removed from mutant PemK by denaturation, a second cycle of Ni-NTA chromatography, and renaturation (Fig. 2A). Ribonuclease assays (Fig. 6A) conducted with $N$. tabacum total RNA as substrate indicated that the same three PemK mutants, defective with respect to growth inhibition (R3A, G16E, and D79V), also lacked ribonuclease activity. Lack of ribonuclease activity of PemK mutants was not

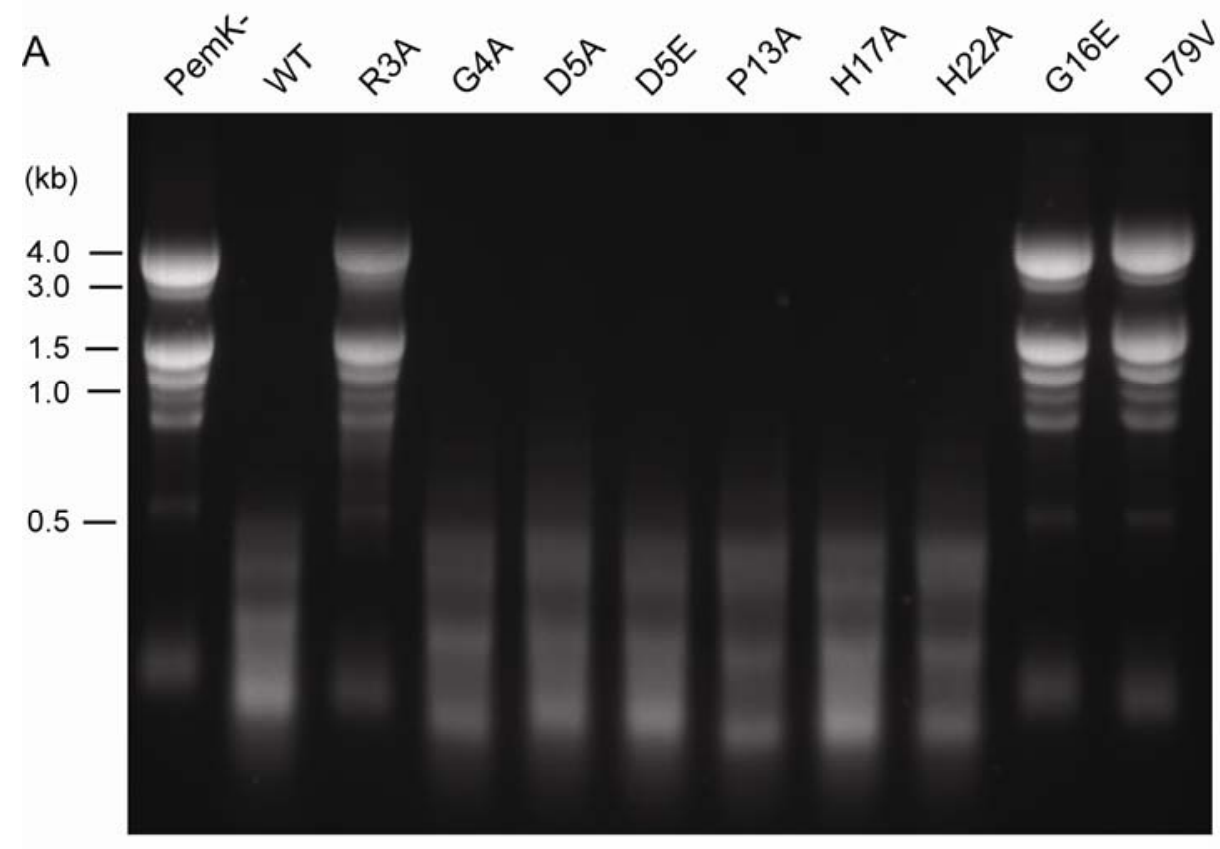

B

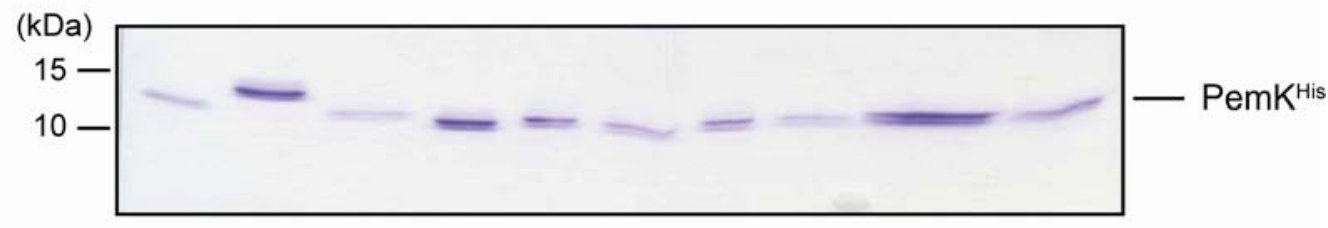

C

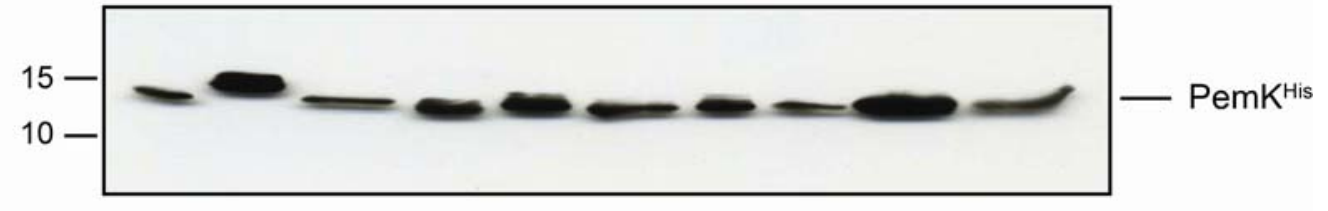

D

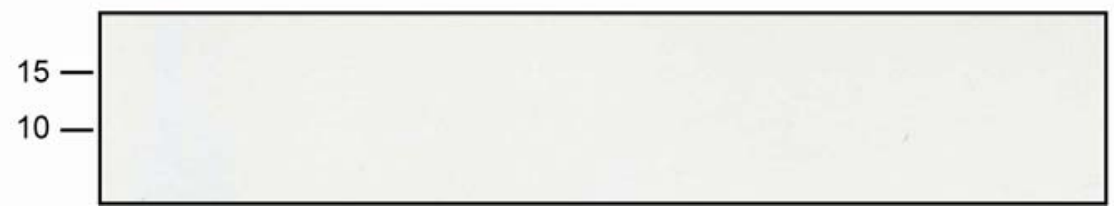

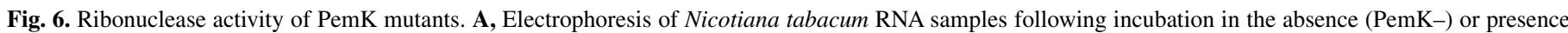

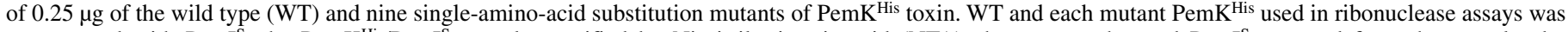

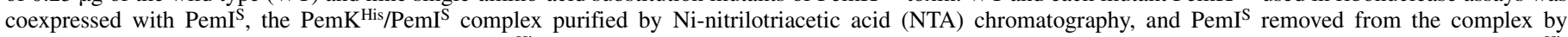

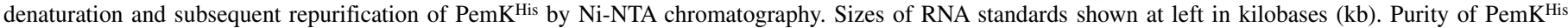

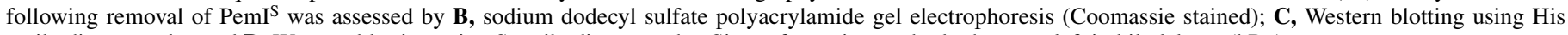
antibodies as probe; and D, Western blotting using $\mathrm{S}$ antibodies as probe. Sizes of protein standards shown at left in kilodaltons (kDa). 
due to the presence of residual PemI, based upon SDS-PAGE (Fig. 6B) and Western blots (Fig. 6C and D).

\section{DISCUSSION}

The pemI/pemK TA system of pXF-RIV11 confers stability of inheritance in the absence of antibiotic selection but the underlying mechanism was unknown. PemK toxin encoded by $X$. fastidiosa pXF-RIV11 was shown to be a potent endoribonuclease able to degrade ssRNA. Several PemK mutants deficient for nuclease activity did not inhibit growth, whereas all nuclease-competent PemK mutants did inhibit growth. Binding of the cognate PemI antitoxin inhibited nuclease activity of PemK, and coexpression of PemI and PemK did not inhibit growth (17). Furthermore, nuclease activity of PemK could be restored by removal of PemI from PemK/PemI complexes. Thus, the mechanism of stable inheritance conferred by the $X$. fastidiosa $\mathrm{pXF}$-RIV11 pemI/pemK TA system is similar to that encoded by plasmid R100 of E. coli (40), even though the two PemK homologues are distantly related and share only $50 \%$ amino acid sequence identity.

Total RNA from N. tabacum was selected as a test substrate for PemK nuclease assays because such samples are simple to prepare and contain a mixture of highly abundant RNAs. PemK of pXF-RIV11 efficiently cleaved deproteinated plant rRNA in vitro. However, in vivo assays of R100 PemK ribonuclease activity indicated that mRNA but not rRNA was cleaved (40), presumably due to inaccessibility of rRNA in highly structured ribonucleoprotein complexes. In vivo, the likely target of pXF-RIV11 PemK also is mRNA. Indeed, a synthetic transcript of one such sequence (cys $G$ ) from $X$. fastidiosa was efficiently cleaved by pXF-RIV11 PemK.

The size range of digestion products suggested that pXF-RIV11 PemK cleaved ssRNA at specific sequences. Sequencing of clones amplified by $5^{\prime}$ RACE consistently indicated a preference for cleavage between $U$ and A residues of UAC, with efficiency of cleavage influenced by identity of the adjacent base 3' proximal to UAC (e.g., U and G preferred over A and C). PemK of E. coli plasmid R100 also preferentially cleaves UAC (40) but no effect of the 3'-proximal base adjacent to UAC has been reported. In contrast, ribonuclease activities of PemK encoded by B. anthracis and $E$. coli mazf are distinct, with preferential cleavage adjacent to pyrimidines (2) or the sequence ACA (39), respectively. R100 PemK also cleaves at a number of alternative sites, including UAA and UAU (39). Alternative recognition sites for pXF-RIV11 PemK were not identified, either because there were none in the targets selected or, if present, cleavage at such sites was inefficient (and not discernible above the background of cDNA termini resulting from premature termination of reverse transcription).

Single-amino-acid substitution mutations introduced into pXFRIV11 PemK were intentionally clustered within the aminoproximal region that contained the conserved motif RGD/E (amino acid residues 3 to 5). Deletion of amino-proximal residues (including the RGD motif) abolished growth inhibition by pXFRIV11 PemK (17). Substitution mutants G4A, D5A, and D5E retained growth inhibition and ribonuclease activity whereas substitution of arginine with alanine (R3A) abolished both phenotypes. These observations confirmed the essential nature of the RGD motif for PemK toxicity.

The amino-proximal region also contains the only histidine residues (positions 17 and 22) present in pXF-RIV11 PemK. These residues were targeted for mutation because histidine participates in acid-base coupling (with glutamine) required for ribonuclease activity of $B$. anthracis PemK (2). Because mutation of either histidine residue did not affect ribonuclease activity, catalysis of RNA degradation by pXF-RIV11 PemK either does not utilize histidine-mediated acid-base coupling or, alternatively, such activity is redundant and may be performed by either histidine residue.
The results presented here provide the first genetic and biochemical characterization of PemK encoded by pXF-RIV11. Although widely spaced mutations (R3A, G16E, and D79V) did abolish ribonuclease (and toxin) activity of pXF-RIV11 PemK, how these mutations interfere with cleavage of RNA is not known. Furthermore, all PemK mutants retained the ability to bind PemI, indicating that domains involved in protein-protein interaction remain to be elucidated.

\section{ACKNOWLEDGMENTS}

We thank A. Spear for providing in vitro RNA transcripts, M. Sisterson for statistical analysis, and K. Zhang for technical assistance. Mention of proprietary or brand names is necessary to report factually on available data; however, the United States Department of Agriculture (USDA) neither guarantees nor warrants the standard of the product, and the use of the name by the USDA implies no approval to the exclusion of others that also may be suitable.

\section{LITERATURE CITED}

1. Afif, H., Allali, N., Couturier, M., and Van Melderen, L. 2001. The ratio between $\mathrm{CcdA}$ and $\mathrm{CcdB}$ modulates the transcriptional repression of the ccd poison-antidote system. Mol. Microbiol. 41:73-82.

2. Agarwal, S., Mishra, N. K., Bhatnagar, S., and Bhatnagar, R. 2010. The PemK toxin of Bacillus anthracis is a ribonuclease: An insight into its active site, structure and function. J. Biol. Chem. 285:7254-7270.

3. Backus, E. A., and Morgan, D. J. W. 2011. Spatiotemporal colonization of Xylella fastidiosa in its vector supports the role of egestion in the inoculation mechanism of foregut-borne plant pathogens. Phytopathology 101:912-922.

4. Bernard, P., and Coutuier, M. 1992. Cell killing by the F plasmid CcdB protein involves poisoning of DNA-topoisomerase II complexes. J. Mol. Biol. 226:735-745.

5. Christensen, S. K., and Gerdes, K. 2003. RelE toxins from bacteria and Archaea cleave mRNAs on translating ribosomes, which are rescued by tmRNA. Mol. Microbiol. 48:1389-1400.

6. Christensen, S. K., Mikkelsen, M., Pedersen, K., and Gerdes, K. 2011. RelE, a global inhibitor of translation, is activated during nutritional stress. Proc. Natl. Acad. Sci. USA 98:14328-14333.

7. Christensen, S. K., Pedersen, K., Hansen, F. G., and Gerdes, K. 2003. Toxin-antitoxin loci as stress-response elements: ChpAK/MazF and ChpBK cleave translated RNAs and are counteracted by mRNA. J. Mol. Biol. 322:809-819.

8. Cooper, T. F., and Heinemann, J. A. 2000. Postsegregational killing does not increase plasmid stability but acts to mediate the exclusion of competing plasmids. Proc. Natl. Acad. Sci. USA 97:12643-12648.

9. Correia, F. F., D’Onofrio, A., Rejtar, T., Li, L., and Karger, B. L. 2006. Kinase activity of overexpressed HipA is required for growth arrest and multidrug tolerance in Escherichia coli. J. Bacteriol. 188:8360-8367.

10. Fineran, P. C., Blower, T. R., Foulds, I. J., Humphreys, D. P., Lilley, K. S., and Salmond, G. P. C. 2009. The phage abortive infection system, ToxIN, functions as a protein-RNA toxin-antitoxin pair. Proc. Natl. Acad. Sci. USA 106:894-899.

11. Fozo, E. M., Hemm, M. R., and Stortz, G. 2008. Small toxic proteins and the antisense RNAs that repress them. Microbiol. Mol. Biol. Rev. 72:579589.

12. Gerdes, K., Chirstensen, S. K., and Lobner-Olesen, A. 2005. Prokaryotic toxin-antitoxin stress response loci. Nat. Rev. Microbiol. 3:371-382.

13. Gerdes, K., Rasmussen, P. B., and Molin, S. 1986. Unique type of plasmid maintenance function: postsegregational killing of plasmid-free cells. Proc. Nat. Acad. Sci. USA 83:3116-3120.

14. Hallez, R., Geeraerts, D., Sterckx, Y., Mine, N., Loris, R., and Van Melderen, L. 2010. New toxins homologous to ParE belonging to threecomponent toxin-antitoxin systems in Escherichia coli O157:H7. Mol. Microbiol. 76:719-732.

15. Hu, M.-X., Zhang, X., Li, E.-L., and Feng, Y.-J. 2010. Recent advancements in toxin and antitoxin systems involved in bacterial programmed cell death. Int. J. Microbiol. Online publication. doi:10.1155/2101/ 781430 .

16. Kolodkin-Gal, I., Verdiger, R., Shlosberg-Fedida, A., and EngelbergKulka, H. 2009. A differential effect of E. coli toxin-antitoxin systems on cell death in liquid media and biofilm formation. PLoS One 8:e6785.

17. Lee, M. W., Rogers, E. E., and Stenger, D. C. 2010. Functional characterization of replication and stability factors of an incompatibility group P-1 plasmid from Xylella fastidiosa. Appl. Environ. Microbiol. 76:7734-7740. 
18. Makarova, K. S., Wolf, Y. L., and Koonin, E. V. 2009. Comprehensive comparative-genomic analysis of type 2 toxin-antitoxin systems and related mobile stress response systems in prokaryotes. Biol. Direct 4:article 19.

19. Matsumoto, A., Young, G. M., and Igo, M. M. 2009. Chromosome-based genetic complementation system for Xylella fastidiosa. Appl. Environ. Microbiol. 75:1679-1687.

20. Moritz, E. M., and Hergenrother, P. J. 2007. Toxin-antitoxin systems are ubiquitous and plasmid-encoded in vancomycin-resistant enterococci. Proc. Natl. Acad. Sci. USA 104:311-316.

21. Pandey, D. P., and Gerdes, K. 2005. Toxin-antitoxin loci are highly abundant in free-living but lost from host-associated prokaryotes. Nucleic Acids Res. 33:966-976.

22. Pedersen, K., Zavialov, A. V., and Pavlov, M. Y. 2003. The bacterial toxin RelE displays codon-specific cleavage of mRNAs in the ribosomal A site. Cell 112:131-140.

23. Prysak, M. H., and Mozdzierz, C. J. 2009.Bacterial toxin YafQ is an endoribonuclease that associates with the ribosome and blocks translation elongation through sequence-specific and frame-dependent mRNA cleavage. Mol. Microbiol. 71:1071-1087.

24. Raines, R. T. 1998. Ribonuclease A. Chem. Rev. 98:1045-1065.

25. Ruiz-Echevarria, M. J., Berzal-Herranz, A., Gerdes, K., and Diaz-Orejas, R. 1991. The kis and kid genes of the parD maintenance system of plasmid R1 form an operon that is autoregulated at the level of transcription by the co-ordinated action of the Kis and Kid proteins. Mol. Microbiol. 5:2685-2693.

26. Ruiz-Echevarria, M. J., Gimenez-Gallego, G., Sabariegos-Jareno, R., and Diaz-Orejas, R. 1995. Kid, a small protein of the parD stability system of plasmid R1, is an inhibitor of DNA replication acting at the initiation of DNA synthesis. J. Mol. Biol. 247:568-577.

27. Sambrook, J., and Russell, D. W. 2001. Molecular Cloning: A Laboratory Manual, 3rd ed. Cold Spring Harbor Laboratory, Cold Spring Harbor, NY.

28. Sat, B., Reches, M., and Engelberg-Kulka, H. 2003. The Escherichia coli mazEF suicide module mediates thymineless death. J. Bacteriol. 185:1803-1807.

29. Sevin, E. W., and Barloy-Hubler, F. 2007. RASTA-Bacteria: A web-based tool for identifying toxin-antitoxin loci in prokaryotes. Genome Biol. 8:R155.
30. Spear, A., Sharma, N., and Flanegan, J. B. 2008. Protein-RNA tethering: The role of poly $(\mathrm{C})$ binding protein 2 in poliovirus RNA replication. Virology 374:280-291.

31. Stenger, D. C., and Lee, M. W. 2011. Phylogeny of replication initiator protein TrfA reveals a highly divergent clade of incompatibility group P1 plasmids. Appl. Environ. Microbiol. 77:2522-2526

32. Stenger, D. C., Lee, M. W., Rogers, E. E., and Chen, J. 2010. Plasmids of Xylella fastidiosa mulberry-infecting strains share extensive sequence identity and gene complement with pVEIS01 from the earthworm symbiont Verminephrobacter eiseniae. Physiol. Mol. Plant Pathol. 74:238-245.

33. Stenger, D. C., Sisterson, M. S., and French, R. 2010. Population genetics of Homalodisca vitripennis reovirus validates timing and limited introduction to California of its invasive insect host, the glassy-winged sharpshooter. Virology 407:53-59.

34. Stenger, D. C., Sisterson, M. S., Krugner, R., Backus, E. A., and Hunter, W. B. 2009. A new Phytoreovirus infecting the glassy-winged sharpshooter (Homalodisca vitripennis). Virology 386:469-477.

35. Stenger, D. C., Young, B. A., and French, R. 2006. Random mutagenesis of Wheat streak mosaic virus HC-Pro: noninfectious interfering mutations in a gene dispensable for systemic infection of plants. J. Gen. Virol. 87:2741-2747.

36. Tsuchimoto, S., Nishimura, Y., and Ohtsubo, E. 1992. The stable maintenance system pem of plasmid R100: Degradation of PemI protein may allow PemK protein to inhibit cell growth. J. Bacteriol. 174:4205-4211.

37. Tsuchimoto, S., Ohtsubo, H., and Ohtsubo, E. 1988. Two genes, pemK and pemI, responsible for stable maintenance of resistance plasmid R100. J. Bacteriol. 170:1461-1466.

38. Wells, J. M., Raju, B. C., Hung, H.-Y., Weisburg, W. G., Mandelco-Paul, L., and Brenner, D. J. 1987. Xylella fastidiosa gen. nov., sp. nov.: gramnegative, xylem-limited, fastidious plant bacteria related to Xanthomonas spp. Int. J. Syst. Bacteriol. 37:136-143.

39. Zhang, Y., Zhang, J., Hoeflich, K. P., Ikura, M., Qing, G., Inouye, M., and Zhu, L. 2003. MazF cleaves cellular mRNAs specifically at ACA to block protein synthesis in Escherichia coli. Mol. Cell 12:913-923.

40. Zhang, J., Zhang, Y., Zhu, L., Suzuki, M., and Inouye, M. 2004 Interference of mRNA function by sequence-specific endoribonuclease PemK. J. Biol. Chem. 279:20678-20684. 